\title{
Metabolically healthy obesity, transition to unhealthy phenotypes, and type 2 diabetes in 0.5 million Chinese adults: the China Kadoorie Biobank
}

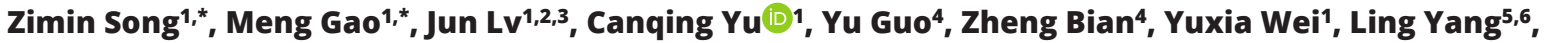 \\ Huaidong Du ${ }^{5,6}$, Yiping Chen ${ }^{5,6}$, Jianqiang Zhang', Jvying Yao ${ }^{8}$, Junshi Chen', Zhengming Chen ${ }^{6}$, Tao Huang ${ }^{1,2}$ \\ and Liming Li' ${ }^{1}$ on behalf of for the China Kadoorie Biobank (CKB) Collaborative Group
}

${ }^{1}$ Department of Epidemiology and Biostatistics, School of Public Health, Peking University Health Science Center, Beijing, China, ${ }^{2}$ Key Laboratory of Molecular Cardiovascular Sciences (Peking University), Ministry of Education, Beijing, China, ${ }^{3}$ Peking University Institute of Environmental Medicine, Beijing, China, ${ }^{4}$ Chinese Academy of Medical Sciences, Beijing, China, ${ }^{5}$ Medical Research Council Population Health Research Unit at the University of Oxford, Oxford, UK, ${ }^{6} \mathrm{Clinical}$ Trial Service Unit \& Epidemiological Studies Unit (CTSU), Nuffield Department of Population Health, University of Oxford, Oxford, UK, ${ }^{7}$ Zhouquan Town Health Center, Tongxiang, Zhejiang, China, ${ }^{8}$ Gaoqiao Town Health Center, Tongxiang, Zhejiang, China, and ${ }^{9}$ China National Center for Food Safety Risk Assessment, Beijing, China

*(Z Song and M Gao contributed equally to this work)

Correspondence should be addressed to T Huang or $\mathrm{H} \mathrm{Du}$ or L Li Email

huangtao@bjmu.edu.cn or huaidong.du@ndph.ox.ac.uk or Imlee@vip.163.com

\begin{abstract}
Objectives: To prospectively assess the association of metabolic health status and its transition with incident diabetes risk across BMI categories.

Design: Cohort study based on the China Kadoorie Biobank (CKB).

Methods: The CKB study enrolled 512715 adults aged 30-79 years from ten diverse areas in China during 2004-2008. After exclusion, 432763 participants were cross-classified by BMI categories and the metabolic status was followed up for incident diabetes disease. The changes in BMI and metabolic health status were defined from baseline to the second resurvey.

Results: Type 2 diabetes risk is higher for metabolically healthy obese (MHO) subjects than metabolically healthy normal weight (MHN) individuals (HR: 3.97, 95\% Cl: 3.64-3.66), and it is highest for those affected by metabolically unhealthy obese (MUO) (HR: 6.47, 95\% Cl: 6.17-6.79). About $15.26 \%$ of participants with MHN converted to metabolically healthy overweight or obesity ( $\mathrm{MHOO}$ ), whereas $48.40 \%$ of $\mathrm{MHOO}$ remained unconverted throughout the follow-up. In obese or overweight people, the conversion from metabolically healthy to unhealthy might increase the chances of developing diabetes as compared to those with a stable metabolic healthy state (HR: 3.70, 95\% Cl: $2.99-$ 4.59), while those with persistent metabolic disorders are most likely to have diabetes (HR: 8.32, 95\% Cl: 7.08-9.78). Conclusions: Metabolic healthy is a transient state, and individuals converted from metabolically healthy status to unhealthy phenotypes across all BMI categories might raise the risk of diabetes.
\end{abstract} Printed in Great Britain

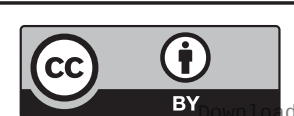

This work is licensed under a Creative Commons Attribution 4.0 International License. 


\section{Introduction}

The worldwide burden of diabetes is enormous and increasing sharply, estimated to be 438 million by 2030 (1). A national survey in China showed that the estimated prevalence of diabetes was 12.8\% in 2017 (2). Obesity and related metabolic disorders have been a well-established causal factor for type 2 diabetes mellitus (T2DM) $(3,4)$. Nevertheless, some obese individuals may not develop metabolic disorders like elevated blood pressure, abnormal lipid profiles, and low insulin sensitivity. This subgroup of obesity displaying a relatively favorable metabolic profile may be referred to as 'metabolically healthy obesity' (MHO) $(5,6)$. Generally, it is estimated that $3-22 \%$ of the general population is affected by $\mathrm{MHO}(7,8)$.

Large-scale epidemiological studies had shown that $\mathrm{MHO}$ adults had a substantially increased risk of developing T2DM compared with metabolically healthy normal-weight (MHN) participants among Europeans, Japanese, and Koreans $(9,10,11,12)$, and little previous literature systematically examined the diabetes risks among individuals stratified by metabolic health status across BMI categories in Chinese people $(13,14)$. Furthermore, it is worth noting that metabolic healthy might be a transient phenotype, and $\mathrm{MHO}$ individuals were more likely to develop metabolic risks $(15,16)$. However, most studies might overlook this issue, and those focused on transition had a small sample size or limited the research population to females $(11,15)$. Therefore, how the diabetes risk is stratified in this particular population, how metabolic risk factors change in different BMI groups in the longterm follow-up, and how the onset of metabolic disorders affects diabetes risk remain to be elucidated. Given the increasing prevalence of obesity in Chinese adults, a better understanding of the health consequences of $\mathrm{MHO}$ phenotypes and their transitions could contribute to public health and clinical practice, as well as promote more efficient diabetes prevention and management strategies.

Therefore, in the present study, we aimed to examine the extent to which the MHO phenotypes, defined using the ATP-III criteria, and their transitions were associated with incident T2DM across BMI categories over the median follow-up of 11.5 years among Chinese adults using data from the China Kadoorie Biobank (CKB).

\section{Methods}

\section{Study design and participants}

CKB was a population-based longitudinal cohort study started in 2004 with 512715 Chinese adults aged 30-79 years recruited from ten sites (five urban and five rural areas) in China. About 25239 (5\%) participants were randomly invited to the resurvey between August 4, 2013, and September 18, 2014. Detailed information on the study design and data collection in CKB had been reported previously $(17,18)$. All participants filled out an interviewer-administered electronic questionnaire, providing detailed information on sociodemographic characteristics, medications, lifestyle and dietary factors, and a written informed consent form. Participants with a history of diabetes $(n=30300)$, stroke $(n=8884)$, coronary heart disease $(n=15472)$ or cancer $(n=2578)$, as well as those with missing data on plasma glucose $(n=8160)$ or BMI $(n=2)$ were excluded from this study. The Ethical Review Committee of the Chinese Center for Disease Control and Prevention (Beijing, China) and the Oxford Tropical Research Ethics Committee, University of Oxford (UK) approved the study.

\section{Measurement of covariates and metabolic status}

Sociodemographic variables (age, sex, education, occupation, marital status, and household income), lifestyle (smoking, alcohol consumption, and physical activity), and dietary factors (fruit, vegetable, and meat intakes) were self-reported at baseline. Height, weight, waist and hip circumferences, and blood pressure were obtained from physical measurements using a standard protocol and validated instruments (19). BMI categories and metabolic status were classified based on Chinese guidelines and a modified definition of the metabolic syndrome described by international harmonized criteria $(20,21,22)$. Cross-classification of BMI categories and metabolic status (healthy/unhealthy) created six groups (Fig. 1). As shown in Fig. 1, participants who had two of the four criteria were considered metabolically unhealthy at baseline. We repeated the primary analysis using triglyceride (TG) and HDL-C data from the second resurvey to reduce the impact of dyslipidemia classification errors at baseline, as dyslipidemia was diagnosed based on selfreported use of statins. Therefore, participants who met three or more of the five criteria were rated as metabolically unhealthy in the second resurvey. We also described the transition between different BMI and the metabolic status during follow-up.

\section{Follow-up for incident diabetes and diabetes mortality}

Cases of diabetes and deaths were ascertained by linking disease and mortality surveillance system and the national 
1.Elevated waist circumference in men $\geq 90 \mathrm{~cm}$ and in women $\geq 85 \mathrm{~cm}$

2.Dyslipidemia

lipid-lowering drugs or;

Triglycerides level $\geq 1.7 \mathrm{mmol} / \mathrm{L}$;

3.Hypertension

$\mathrm{SBP} \geq 130 \mathrm{mmHg}$ or $\mathrm{DBP} \geq 85 \mathrm{mmHg}$ or;

self-reported hypertension or;

anti-hypertensive drugs;

4.Elevated plasma glucose

fasting glucose $>5.6 \mathrm{mmol} / \mathrm{L}$ and;

fasting glucose $<7.0 \mathrm{mmol} / \mathrm{L}$

Low HDL cholesterol(only second re-survey)

5 .in men $<1.0 \mathrm{mmol} / \mathrm{L}$ and in women $<1.3 \mathrm{mmol} / \mathrm{L}$

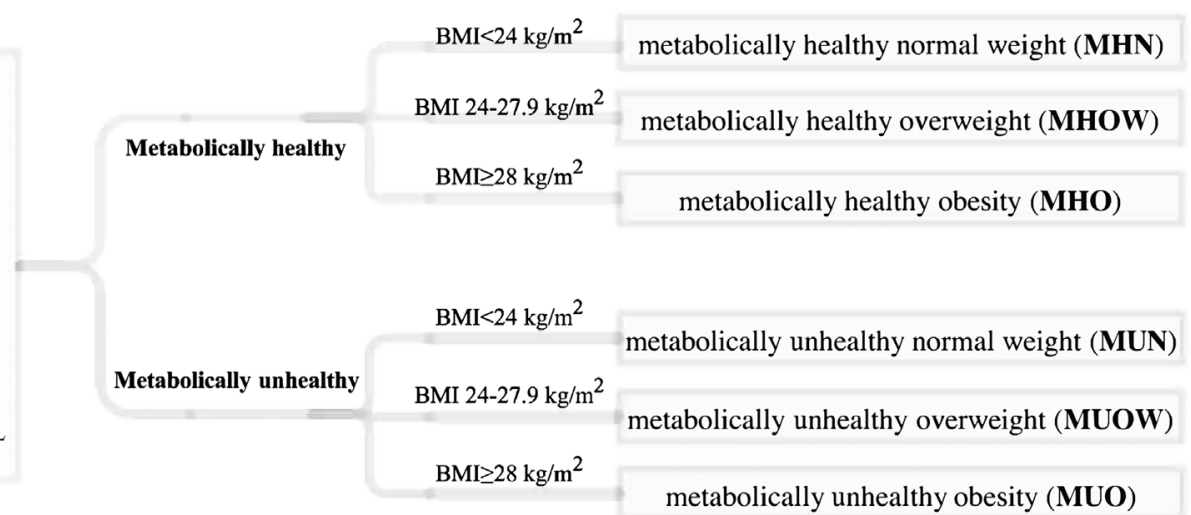

\section{Figure 1}

Definition of metabolically healthy obesity and their transition. SBP, systolic blood pressure; DBP, diastolic blood pressure.

health insurance claim database, with almost universal coverage across the ten study areas (>98\%), supplemented by local residential records and annual active verification. The causes of death were determined primarily by reviewing death certificates, as well as medical records and verbal autopsies using validated instruments. Annual active follow-up was performed by checking against local residential records to confirm survival status and to minimize follow-up losses. More details had been reported elsewhere (23).

All events were coded according to the International Classification of Diseases, 10th Revision (ICD-10) by trained staff blinded to baseline information. Diagnoses of total diabetes are coded according to the International Classification of Diseases, 10th Revision (ICD-10; diabetes: E10-E14). Total diabetes contains insulin-dependent (E10), non-insulin-dependent (E11), malnutrition-related (E12), other specified (E13), and unspecified (E14). The primary outcomes in the present study were type 2 diabetes mellitus (E11) and total diabetes (E10-E14).

\section{Statistical analysis}

Baseline and resurvey sample characteristics are presented as mean (S.D.) or percentage when appropriate. The ANOVA test was used to examine differences between groups in baseline characteristics for continuous variables and the $\chi^{2}$ test for dichotomous measures. The Cox proportional hazard regression model with a time-varying exposure was used to examine the relationship between six phenotypes of $\mathrm{MHO}$ and their transition associated with the risk of diabetes over the follow-up. Person-time was calculated from the date of birth to diabetes diagnosis, death, or the end of follow-up (Dec 31, 2016), whichever came first. Proportional hazards assumption was tested using
Schoenfeld residuals. 95\% CIs were estimated by the logrank test (24). To account for the competing risks of death, competing-risks regression models based on Fine-Gray model were fitted to estimated sub-hazard ratios of $\mathrm{MHO}$ to address the potential competing risk problem from mortality (25).

MHN individuals were used as a reference group in the primary analysis. The main analyses were first adjusted for age, sex, study region, and then further for education, household income, marital status, smoking status, alcohol intake, physical activity, fruit, vegetable and red meat intakes, and family history of diabetes. In categorical analyses, participants were divided into six groups according to MHO phenotypes. HRs were calculated relative to the MHN group and plotted against the MHO six phenotypes. To test whether the pattern of association varies across stratifications, we estimated multiplicative interactions by including the product term (exposure $\times$ stratification variable) in the models using the likelihood ratio test. During the transition analysis, we used stable MHN during the follow-up as the referent group; HRs were also calculated for MHN to metabolically healthy overweight or obesity (MHOO) group, MHOO throughout the group, MHOO to metabolically unhealthy overweight or obesity (MUOO), and MUOO throughout the group.

A subgroup analysis based on sex or age then allowed us to compare the incidence of T2DM among the different groups. Sensitivity analyses were undertaken to assess the impact of excluding cases that had been smokers or occurred in the first 2 years of follow-up or using waist-hip ratio instead of waist circumference to demonstrate the robustness of our results. We also investigated whether results were similar when the metabolic syndrome is defined using the harmonized International Diabetes 
Federation definition, which excludes waist circumference. Two-sided $P<0.05$ was considered statistically significant. All statistical analyses were performed using Stata (version 15.0, StataCorp) and SAS version 9.4 (SAS Institute Inc., Cary, NC, USA).

\section{Results}

\section{Characteristics of the CKB participants}

Of 432763 participants in this analysis, the mean age at baseline was 51.0 (10.4) years, mean BMI was 23.8 (3.1) $\mathrm{kg} / \mathrm{m}^{2}, 177317$ (41.0\%) were men, and 188072 (43.5\%) resided in urban areas (Table 1). Also, 4571 (1.06\%) of 432763 participants failed to follow up, and the rate was not significantly different between the MHO groups after adjusted age, sex, and region $(P$ for trend $=0.291$ ) (data not shown). More than half of the participants (53.4\%) had metabolically healthy normal weight, and only $3.5 \%$ were $\mathrm{MHO}$, in which more women than men (4.1\% vs $2.6 \%$ ) (Fig. 2 and Supplementary Fig. 1, see section on supplementary materials given at the end of this article). The prevalence of participants with metabolically unhealthy normal weight (MUN), metabolically unhealthy overweight (MUOW), and metabolically unhealthy obesity (MUO) was $2.7,9.4$, and $6.8 \%$, respectively (Fig. 2). Mean age and SBP were higher among metabolically unhealthy individuals across all BMI categories than their healthy counterparts. In other words, individuals with $\mathrm{MHO}$ were more likely to be female, younger, living in rural areas, have less physical activity, and have more meat intake than MHN.

\section{Incident diabetes risk in different metabolic health status}

During a median follow-up of 10.1 years, we documented 14 631 cases of total diabetes and $9033 \mathrm{~T} 2 \mathrm{DM}$. In the same BMI category group, individuals with an unhealthy metabolism are more likely to develop T2DM. In comparison with MHN groups, HRs for MUN individuals were 3.00 (95\% CI: 2.74-3.29), 4.49 (95\% CI: 4.29-4.70) for MUOW individuals, and 6.47 (95\% CI: 6.17-6.79) for MUO individuals (Table 2). In metabolically healthy individuals, being overweight or obese still increases the risk of T2DM. Individuals with metabolically healthy overweight (MHOW) (HR: 2.38, 95\% CI: 2.29-2.48) and MHO (HR: 3.97, 95\% CI: 3.62-4.36) are much more susceptible to diabetes than MHN individuals. As for total diabetes, this effect was also observed but weakened.
Furthermore, obese and metabolically unhealthy participants simultaneously are at greater risk of T2DM death (HR for MHOW: 2.05, 95\% CI: 1.31-3.22; HR for MUO: 2.73, 95\% CI: 1.64-4.55). Adjusted for competing risk of other causes of death, there still was a positive correlation between MUO and T2DM mortality (subdistribution HR: 3.54, 95\% CI: 1.89-6.60). For total diabetes death, the standard Cox regression model may have also underestimated the actual strength of the association between $\mathrm{MHO}$ and total diabetes death (subdistribution HR: 3.32 , 95\% CI: $2.13-5.18$ vs HR: 2.59 , 95\% CI: $1.78-3.76$ ) (Supplementary Table 4).

On the basis of gender stratification, the six MHO phenotypes posed a slightly higher total diabetes and T2DM risk among women than men, indicating significant modification effects of sex on the associations ( $P$ for interaction <0.001) (Fig. 3 and Supplementary Fig. 2). When stratified by age groups (30-49 years as the reference group), risks for total diabetes and T2DM gradually increased with aging within each $\mathrm{MHO}$ group $(P$ for interaction $<0.001$ ) (Supplementary Fig. 3). MHO individuals were more prone to develop diabetes than those with normal weight within each age group, while individuals with unhealthy metabolism were most susceptible.

\section{Sensitivity analyses}

In sensitivity analyses, excluding participants who never smoked or cases within the first 2 years after baseline, results were not materially altered. Using a definition of metabolic syndrome that did not contain waist circumference produced enhanced results for MHO; however, with only $0.3 \%$ (45 of 14 631) of participants categorized as $\mathrm{MHO}$ at baseline, few participants were left to transition to the unhealthy state. We also found a more significant association using waist-hip ratio instead of waist circumference (Supplementary Table 3).

\section{The transition of metabolic health status and its association with incident diabetes risk}

About 21890 (5.1\%) of baseline participants were randomly invited to conduct the second resurvey. After exclusion criteria, 7355 (39.4\%) of 18661 participants had the transition of metabolic health status in the second resurvey. The sample size of each MHO subgroup for transition is presented in a $6 \times 6$ contingency table (Supplementary data and Supplementary Table 2). According to the resurvey data, $15.26 \%$ of the 9763 participants who had MHN at baseline switched to MHOO phenotypes, while 


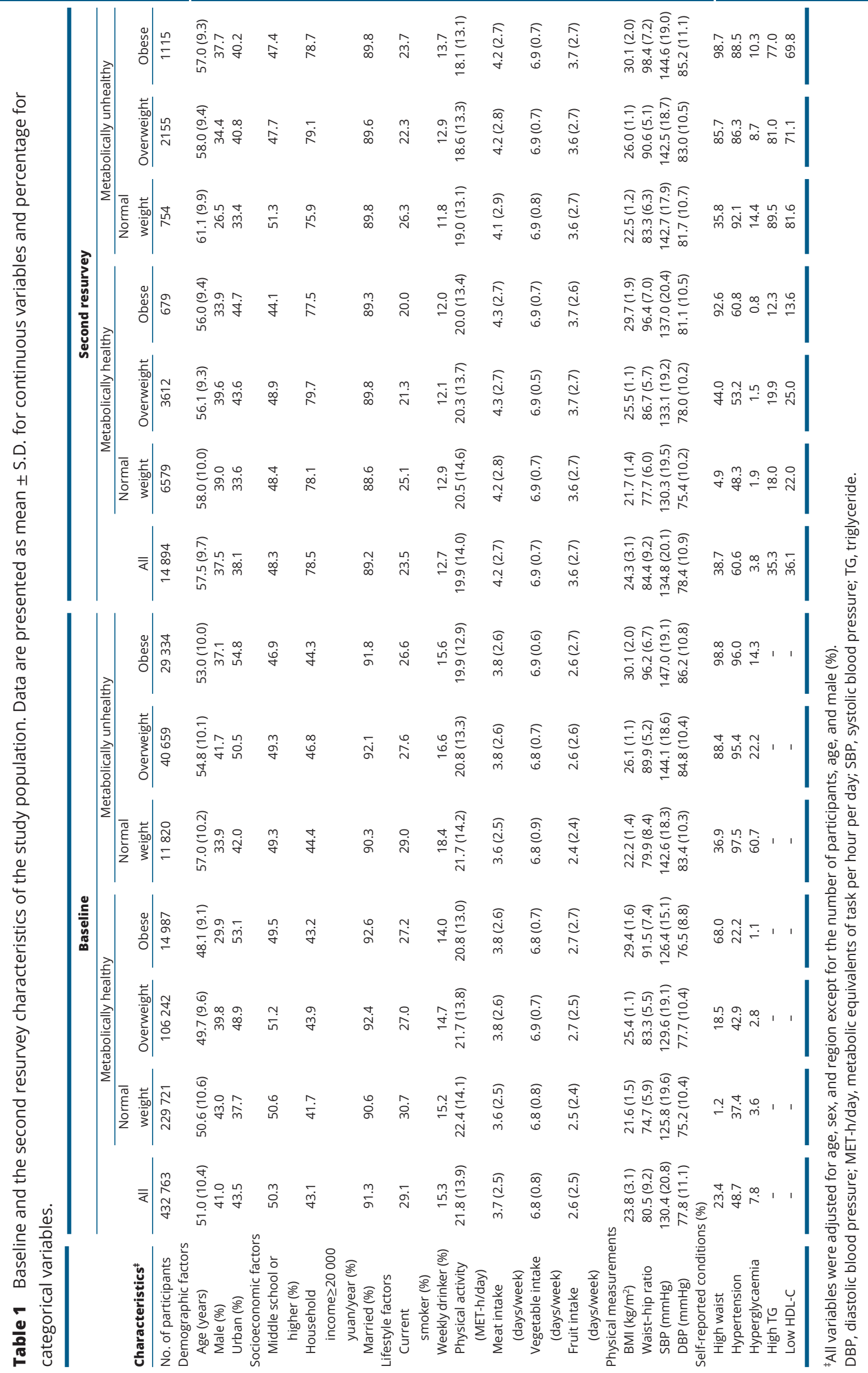


$67.34 \%$ did not. Out of 5802 participants with MHOO, $39.47 \%$ converted to MUOO and $48.40 \%$ failed to convert. However, the majority of MUOO (66.48\%) remained unconverted throughout the follow-up (Fig. 4).

The cumulative incidence of T2DM was higher in participants who changed from metabolic healthy overweight or obesity to MUOO (HR: 3.70, 95\% CI: 2.994.59) compared to stable MHOO participants (HR: 1.05, 95\% CI: 0.72-1.52) but lower than those of stable MUOO participants (HR: 8.32, 95\% CI: 7.08-9.78) who had the highest risk among all groups (Fig. 5). For the participants with metabolic healthy normal weight, the risk of T2DM from MHN to obesity was not significant (HR: 0.85, 95\% CI: 0.48-1.50) but markedly lower than those who maintained MHOO or converted to MUOO (Fig. 5). Then, we utilized the logistic regression with the sex or multivariableadjustment models instead of Cox proportional hazards models to analyze the association between MHO transition from the baseline to the second resurvey and diabetes. Compared with participants who maintained MHN, those who were metabolically unhealthy overweight or obesity throughout baseline and the second resurvey had multivariable-adjusted OR for total diabetes of 8.60 (95\% CI: 6.78-10.91) and type 2 diabetes of 10.85 (95\% CI: 8.0414.63) (Supplementary data and Supplementary Table 5).

\section{Discussion}

In this prospective study of 432763 Chinese adults, our findings show that $\mathrm{MHO}$ individuals are at an increased risk of incident diabetes compared with MHN individuals over a median of 10.1 years of follow-up, implying that obesity is still the leading risk factor for diabetes and further support should be provided for the need for all individuals to maintain a healthy weight. Besides, metabolically unhealthy individuals had a substantially higher risk of diabetes than individuals without existing metabolic conditions across all BMI groups, supporting strategies to prevent metabolic risk factors irrespective of weight status. Likewise, the transition from healthy to unhealthy overweight or obesity and stable unhealthy overweight or obesity substantially increased the risk of incident diabetes.

Our findings in this prospective cohort study are in line with previous results in Western populations $(12,15)$. The Whitehall II study among 7122 participants (69.7\% men), aged 39-63 years, showed that MHO subjects were at increased risk for type 2 diabetes (HR: 3.25, 95\% CI: 2.324.54) compared with MHN individuals over the median
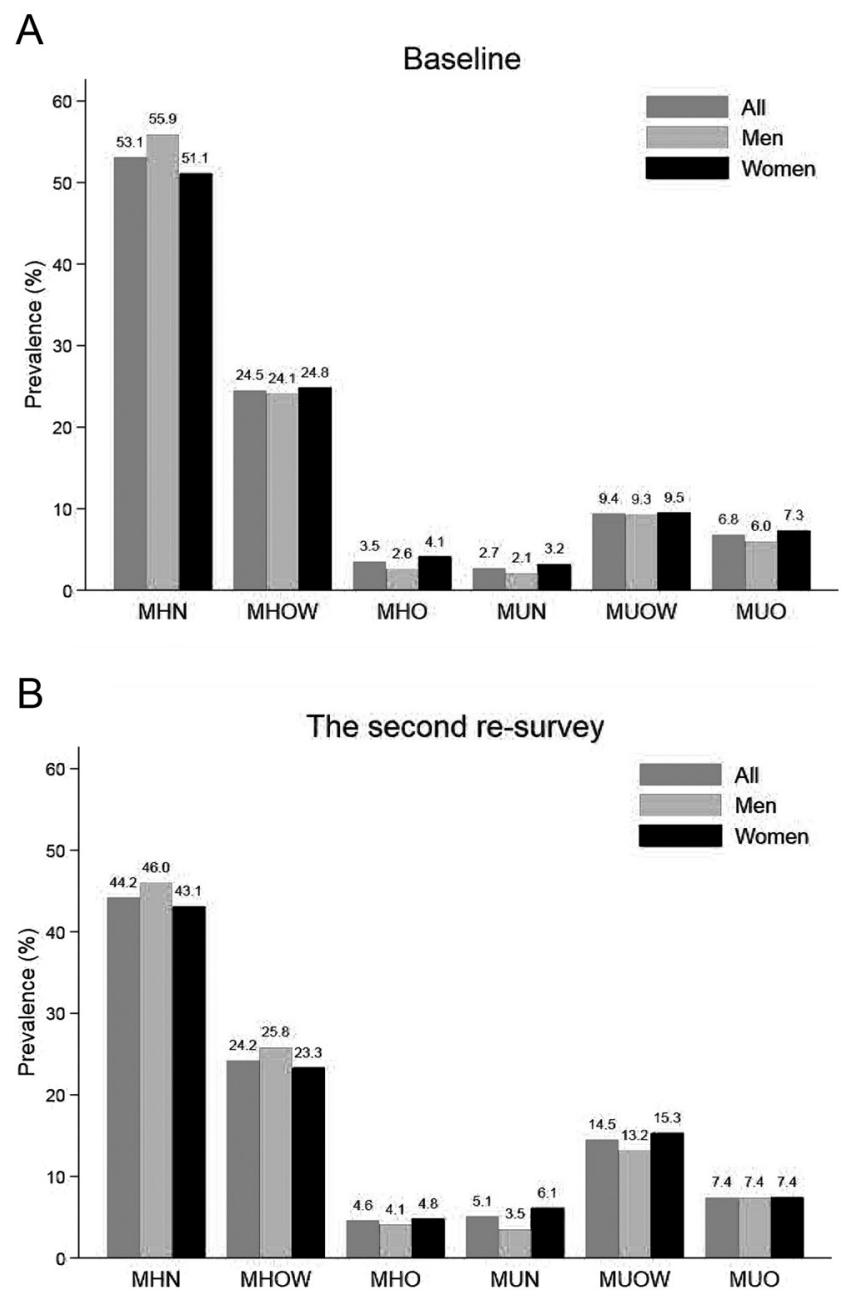

Figure 2

Sex-specific prevalence of $\mathrm{MHO}$ at baseline and second resurvey. ${ }^{\dagger}$ Prevalence is standardized for age and region. $\mathrm{MHN}$, metabolically healthy normal weight; $\mathrm{MHO}$, metabolically healthy obesity; MHOW, metabolically healthy overweight; MUN, metabolically unhealthy normal weight; MUO, metabolically unhealthy obesity; MUOW, metabolically unhealthy overweight.

follow-up of 17.4 years (12). The hazard risk was relatively lower than in our study of 0.5 million Chinese adults (HR: 3.97). Similarly, a meta-analysis of three prospective cohort studies including 134667 East Asian subjects showed that $\mathrm{MHO}$ adults had a distinctly higher risk of developing type 2 diabetes (HR: 3.28, 95\% CI: 2.30-4.67) compared with MHN adults (9), which was similar to the effect size in the Western population, but also lower than that in Chinese adults. Additionally, our study based on large-scale prospective research demonstrated that the HRs for T2DM in $\mathrm{MHO}$ phenotypes were relatively more significant in females than males. Together with previous literature 
8

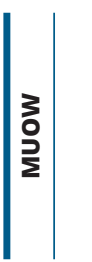

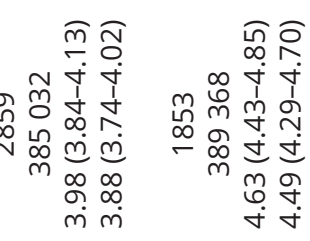

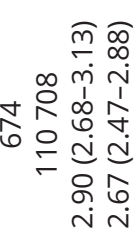

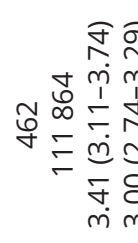

ชิ

L

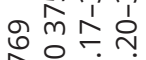

윰

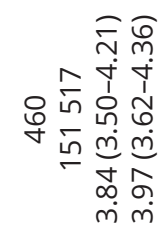

奋

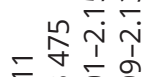

ॠ

$-\infty \bar{o}$
i

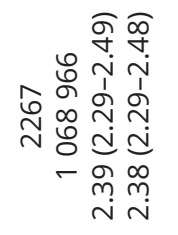

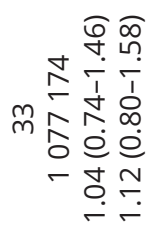

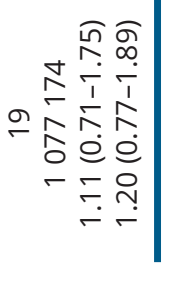

ํํำ

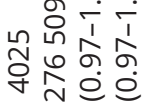

$\sim 8$.

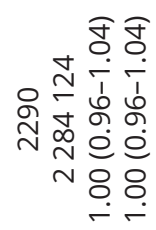

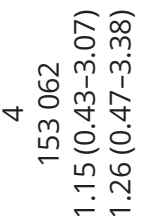

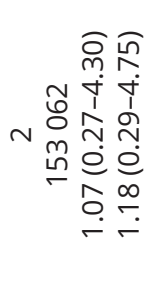

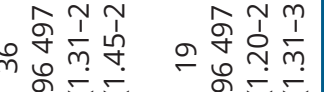

둥

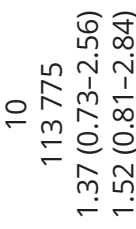

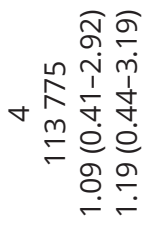

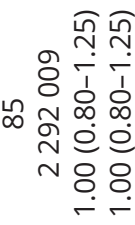

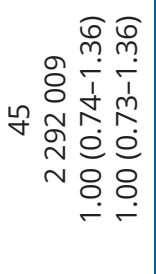

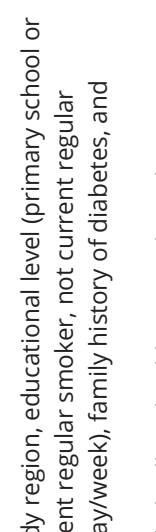

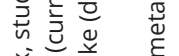

र

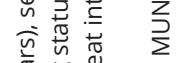

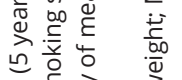

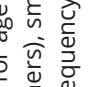

웡

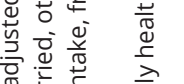

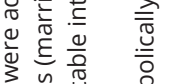

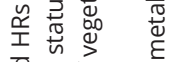

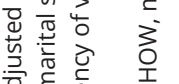

产产焉

苋茎造苟

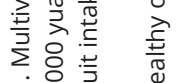

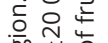

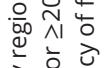

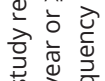

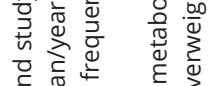

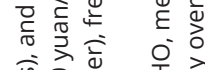

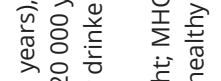

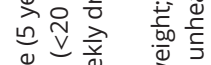

离

\% है $\frac{0}{3} \quad \frac{3}{0}$

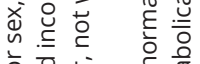

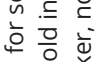

क्ष

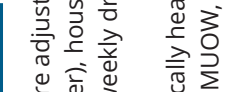

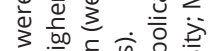

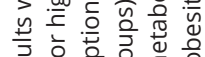

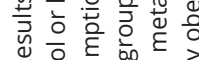

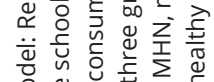

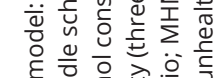

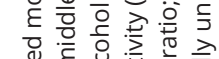




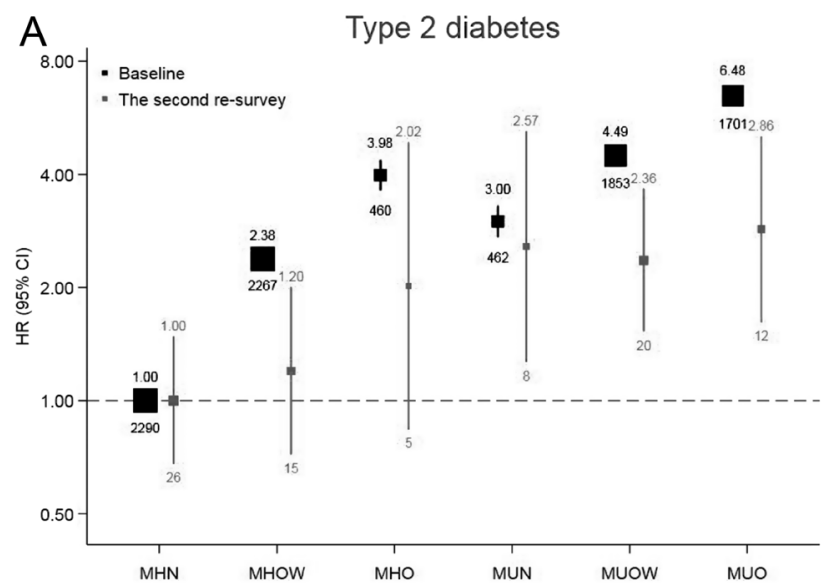

B

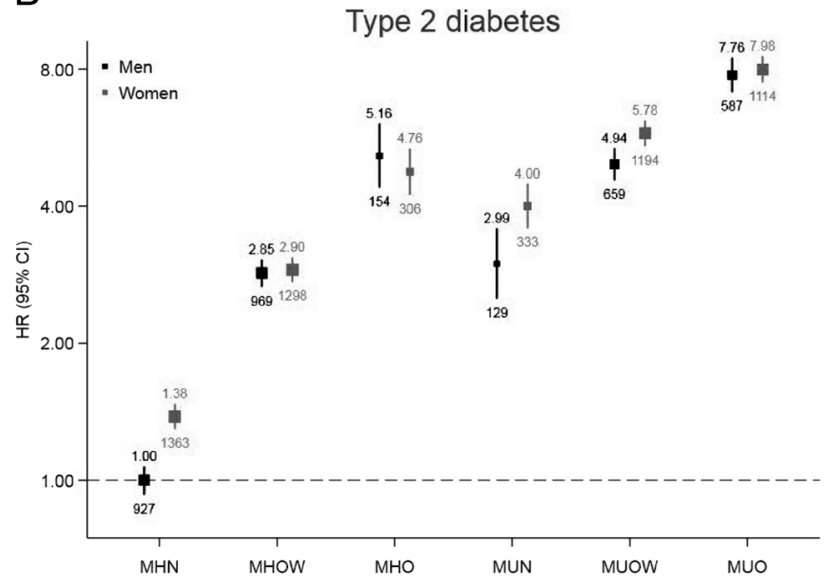

Figure 3

The second resurvey and sex-specific hazard ratios of $\mathrm{MHO}$ for type 2 diabetes. ${ }^{\dagger}$ HRs were adjusted for age (5 years), study region, educational level (primary school or lower and middle school or higher), household income (<20 000 yuan/year or $\geq 20000$ yuan/year), marital status (married, others), smoking status (current regular smoker, not current regular smoker), alcohol consumption (weekly drinker, not weekly drinker), frequency of fruit intake, frequency of vegetable intake, frequency of meat intake (days/week), family history of diabetes and physical activity (three groups). ${ }^{\ddagger} \mathrm{HRs}$ are indicated above the squares, and the number of cases in each category is displayed below the squares. Corresponding 95\% $\mathrm{Cls}$ are plotted as lines. The size of the squares is inversely proportional to the variance of the logarithm of HR. *The relation with $\mathrm{MHO}$ differed by sex for type 2 diabetes ( $P$ for interaction $<0.001)$. MHN, metabolically healthy normal weight; $\mathrm{MHO}$, metabolically healthy obesity; MHOW, metabolically healthy overweight; MUN, metabolically unhealthy normal weight; MUO, metabolically unhealthy obesity; MUOW, metabolically unhealthy overweight; HR, hazard ratio. reports, our findings do not support the notion that healthy obesity is a harmless condition, indicating that the current definitions of metabolic health are insufficient to identify a subgroup of obesity that is not at risk of T2DM $(26,27)$.

It is worth noting that we used Chinese-specific BMI and waist circumference, which is different from Western standards. Previous studies proved that Asian populations at a lower BMI than White populations have a high incidence rate of type 2 diabetes (28). Asian participants are more likely to have been allocated to the normal BMI group if using the same BMI cut-off points for all races. Hence, WHO recommended lower BMI cut-offs for defining obesity in south Asian populations to optimize the early identification of cardiometabolic risk. To better demonstrate the contrast between different BMI cut-off points in different people, some studies had included different ethnic groups to observe the relationship between different BMI cut-off points and glucose factors or the onset of diabetes after adjusting the same covariates $(29,30)$.

Although the MHO group had a higher risk of diabetes than the MHN, as reported previously (11), their risk was significantly lower than that in the MUO (12). At the same time, individuals with MUN had a higher risk of diabetes than $\mathrm{MHN}$, indicating that a normal weight may not mean a healthy metabolic status. That is, normalweight individuals may also be metabolically unhealthy (31). The potential mechanism is that metabolic risk in normal-weight subjects may be more strongly linked to a relatively low leg fat mass than high s.c. abdominal fat (32). This MUN phenotype also exhibits impaired insulin secretion capacity and insulin resistance, insufficient cardio-respiratory fitness, and increased carotid intimamedia thickness (cIMT). In addition, genetic pathways of metabolic risk appear to be different between normalweight and obese individuals $(33,34)$. It is necessary to follow the medical guideline-based recommendations, including the adoption of a healthy lifestyle and the initiation of pharmacological treatments for metabolic risk factors. Since fat mass and fat distribution differ between normal-weight and obese subjects, it is important to take targeted measures promoting fat transformation to reduce metabolic diseases risks $(35,36)$.

In addition, the effect on diabetes differs when a person is only obese or metabolically unhealthy. MHO individuals were at an increased risk of incident diabetes compared with MUN individuals with East Asian studies (9) but not with Western research results (12). For example, the Whitehall II cohort study among 7122 participants showed that MHO and MUN groups were at a similar increased 


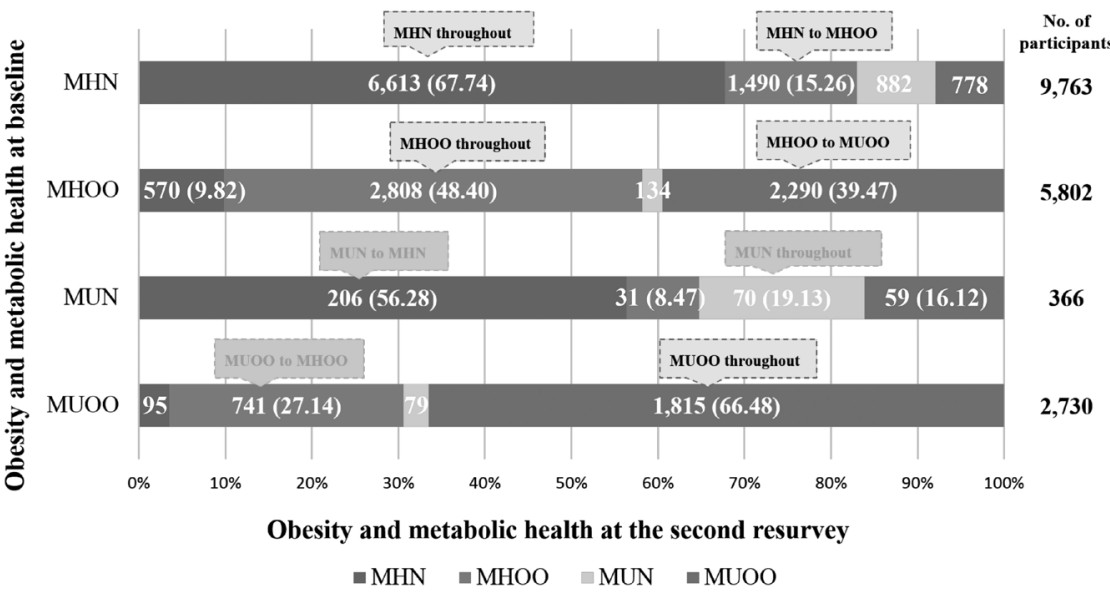

\section{Figure 4}

Transition of MHO status from baseline to the second resurvey. MHN, metabolically healthy normal weight; $\mathrm{MHOO}$, metabolically healthy overweight or obesity; MUN, metabolically unhealthy normal weight; MUOO, metabolically unhealthy overweight or obesity.

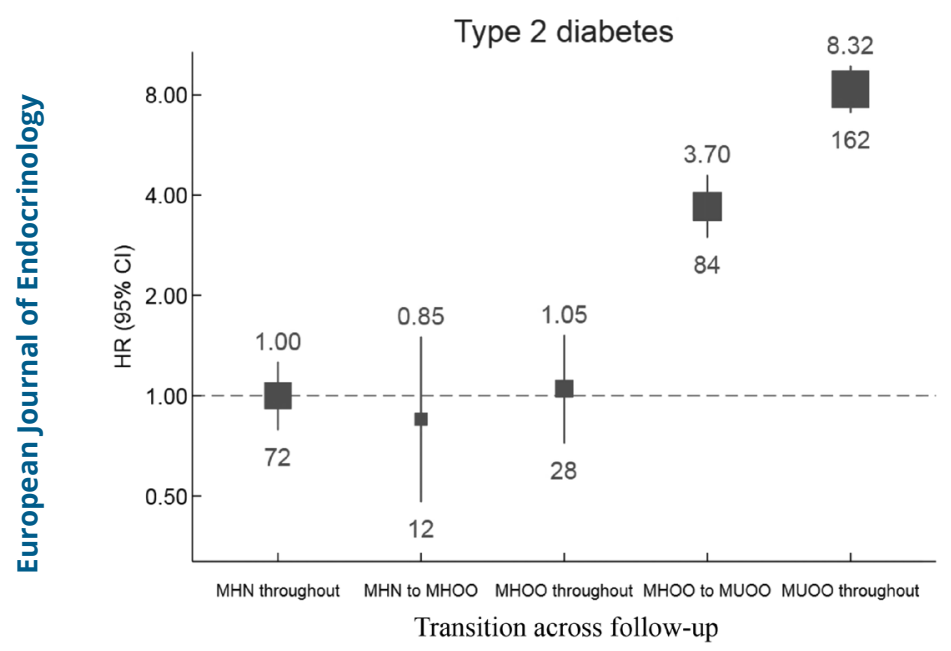

\section{Figure 5}

Transition between metabolic status and association with diabetes risk. ${ }^{\dagger} \mathrm{HRs}$ were adjusted for age (5 years), sex, study region, educational level (primary school or lower and middle school or higher), household income ( $<20000$ yuan/year or $\geq 20000$ yuan/year), marital status (married, others), smoking status (current regular smoker, not current regular smoker), alcohol consumption (weekly drinker, not weekly drinker), frequency of fruit intake, frequency of vegetable intake, frequency of meat intake (days/week), family history of diabetes, and physical activity (three groups). ${ }^{\ddagger} \mathrm{HRs}$ are indicated above the squares, and the number of cases in each category is displayed below the squares. Corresponding 95\% $\mathrm{Cls}$ are plotted as lines. The size of the squares is inversely proportional to the variance of the logarithm of HR. HR, hazard ratio; MHN, metabolically healthy normal weight; $\mathrm{MHOO}$, metabolically healthy overweight or obesity; MUOO, metabolically unhealthy overweight or obesity. risk of incident type 2 diabetes (12). The discrepancy of such association may be due to ethnic differences and the much smaller sample size but longer follow-up in the Western study.

Metabolic health might be a transient state for most obese individuals $(15,16,37)$. In contrast to previous studies, which only examined the association of metabolic health at baseline with the risk of diabetes $(9,10,13,14)$, we addressed transition in obesity or metabolic health status over time by reviewing the health status at baseline and resurvey. Most metabolically healthy participants (67.74\%) still maintain normal weight; only a small proportion (15.26\%) of participants with initial MHN converted to MHOO after 10 years of follow-up. Similarly, people with overweight or obesity have a greater tendency to transit from metabolically healthy to metabolically unhealthy states. Whitehall II cohort study showed that about half of the participants with initial MHO converted to MUO over 20 years (38) which was somewhat higher than our study in Chinese adults (39.53\%). The Nurses' Health Study among 90257 women indicated that only $6.1 \%$ of MHO were still metabolically healthy over 30 years (15). All participants were women, and a longer follow-up duration in the Nurses' Health Study could explain the observed higher conversion rates (15).

However, no study investigated the association of the transition of individuals' metabolic health with the risk of diabetes over a longer follow-up to our knowledge. In the present study, the high conversion rates (39.47\%) from metabolic healthy to unhealthy obesity indicate that singlepoint determination of metabolic health is probably not appropriate to establish long-term diabetes risk precisely. Significantly, overweight or obese participates with stable metabolically unhealthy status showed a substantially higher risk of developing diabetes, which was two-fold higher than overweight or obese participants who changed 
from metabolically healthy to unhealthy situations. Based on the current study results, metabolic healthy obese alone cannot always be regarded as a stable characterization of lower clinical risk. Yet, it's worth noting that only around $5 \%$ of the baseline studied population had measurements at the second resurvey. It is a fact that the existence of sampling error is inevitable. Hence, we conclude that the prevalence of metabolic status needs to be reviewed critically. Future studies should pay more attention to the transition of metabolic health and its increased risk for diabetes.

The strengths of the current study include the large number of diabetic cases, the prospective design, the repeated measurements, its long follow-up time, the high quality of cardiometabolic risk factors measurement, and the implementation of different sensitivity analyses. Further, all participants were recruited at random and consecutively from the general population; thus, the potential for selection bias is minimal. Notably, we considered the transition of metabolic health over time in estimating the risk of diabetes specifically among individuals with obesity $(15,39)$; therefore, the accuracy of diabetes risk estimates might have been improved. For our observational studies, however, the results must be interpreted with caution. There are also some limitations that should be mentioned. First, as the study population consisted of Chinese adults, the generalizability to other ethnic groups is limited. Secondly, similar to the definition of metabolic health in the previous study (26), we considered anti-dyslipidemia drugs in defining metabolic status, which may not rule out that stricter definitions of metabolic health components would identify a healthier subgroup. Thirdly, although we validated our results in resurvey data, misclassification of metabolic health which might bias the observed associations cannot be excluded. Fourthly, we adjusted for cardiometabolic risk factors, but residual confounding remains possible as generally in observational studies. Fifthly, as diabetes was the outcome of interest, we further excluded participants with missing baseline glucose data to minimize potential reverse causality bias for those who might have undiagnosed diabetes. The incidence density of diabetes and diabetes mortality was higher among participants with missing glucose data (Supplementary data and Supplementary Table 1). This strict exclusion criterion may have introduced selection bias, leading to underestimation of diabetes and diabetes mortality incidence rate.

In conclusion, overweight or obese participants with and without metabolic syndrome are associated with an increased risk of diabetes in the general population. Our results also reinforce recommendations that maintaining metabolic health in all BMI groups for diabetes prevention may be helpful in China. Moreover, metabolically healthy status as a transient state was shown to far increase the risk of type 2 diabetes when it changed to a metabolically unhealthy situation. Particular attention should be given to the impact of metabolic health and how it changes over time increasing the risk of diabetes risk.

\section{Supplementary materials}

This is linked to the online version of the paper at https://doi.org/10.1530/ EJE-21-0743.

\section{Declaration of interest}

All authors declare no support from companies for the submitted work; no relationships with companies that might have an interest in the submitted work in the previous 3 years; no spouses, partners, or children that have financial relationships that may be relevant to the submitted work; and no non-financial interests that may be relevant to the submitted work.

\section{Funding}

This work was supported by grants (2020YFC2003401, 2016YFC0900500, 2016YFC0900501, 2016YFC0900504) from the National Key R\&D Program of China. The CKB survey was supported by grants from the Kadoorie Charitable Foundation in Hong Kong, National Natural Science Foundation of China (81390540, 81390544, 81390541), and the Chinese Ministry of Science and Technology (2011BAI09B01). Funders have no role in research design, data collection, data analysis and interpretation, report writing or submission decisions for publication.

\section{Availability of data and material}

Data are available in a public, open access repository. This research has been conducted using the China Biobank Resource under Application Number 2018-0066. The China Biobank data are available on application to the China Biobank (https://www.ckbiobank.org/site/).

\section{Ethics approval}

The Ethical Review Committee of the Chinese Center for Disease Control and Prevention (Beijing, China) and the Oxford Tropical Research Ethics Committee, University of Oxford (UK), approved the study.

\section{Consent to participate}

All participants consent to participate in the present study.

\section{Consent for publication}

Consent for publication was obtained from all authors.

\section{Author contribution statement}

Conceptualization was done by Canqing Yu, Yiping Chen, Zhengming Chen, Tao Huang, Liming Li. Data curation was done by Zimin Song, Meng 
Gao, Jun Lv, Canqing Yu, Yu Guo, Zheng Bian, Yuxia Wei, Huaidong Du, Ling Yang, Zhengming Chen, Liming Li. Formal analysis was done by $\mathrm{Yu}$ Guo, Yuxia Wei, Huaidong Du, Ling Yang, Yiping Chen, Jianqiang Zhang, Jvying Yao, Junshi Chen, Tao Huang, Liming Li. Funding acquisition was done by Liming Li. Investigation was done by Zhengming Chen, Tao Huang, Liming Li. Zimin Song, Meng Gao, Jun Lv, Zheng Bian, Yuxia Wei, Zhengming Chen provided the methodology. The original draft was written by Zimin Song, Meng Gao, Yu Guo, Zheng Bian, Yuxia Wei, Huaidong Du, Ling Yang, Yiping Chen, Jianqiang Zhang, Jvying Yao, Junshi Chen, Zhengming Chen, Tao Huang, Liming Li. The writing was reviewd and edited by Tao Huang, Liming Li.

\section{Acknowledgements}

The authors thank all the participants and staff of this study and each survey team and management team member for their support.

\section{References}

1 Hu FB. Diet and exercise for new-onset type 2 diabetes? Lancet 2011 378 101-102. (https://doi.org/10.1016/S0140-6736(11)60692-2)

2 Li Y, Teng D, Shi X, Qin G, Qin Y, Quan H, Shi B, Sun H, Ba J, Chen B et al. Prevalence of diabetes recorded in mainland China using 2018 diagnostic criteria from the American Diabetes Association: national cross sectional study. BMJ 2020369 m997. (https://doi.org/10.1136/bmj.m997)

3 Lyall DM, Celis-Morales C, Ward J, Iliodromiti S, Anderson JJ, Gill JMR, Smith DJ, Ntuk UE, Mackay DF, Holmes MV et al. Association of body mass index with cardiometabolic disease in the UK Biobank: a Mendelian randomization study. JAMA Cardiology 20172 882-889. (https://doi.org/10.1001/jamacardio.2016.5804)

4 Kahn SE, Hull RL \& Utzschneider KM. Mechanisms linking obesity to insulin resistance and type 2 diabetes. Nature $2006 \mathbf{4 4 4} 840-846$. (https://doi.org/10.1038/nature05482)

5 Stefan N, Häring HU, Hu FB \& Schulze MB. Metabolically healthy obesity: epidemiology, mechanisms, and clinical implications. Lancet: Diabetes and Endocrinology 20131 152-162. (https://doi.org/10.1016/ S2213-8587(13)70062-7)

6 Schulze MB. Metabolic health in normal-weight and obese individuals. Diabetologia 201962 558-566. (https://doi.org/10.1007/ s00125-018-4787-8)

7 Zhang M, Tong W, Chen J, Zhang Y \& Li S. Metabolically healthy obesity and its associates in Mongolian Chinese adults. Metabolic Syndrome and Related Disorders 201412 185-190. (https://doi. org $/ 10.1089 /$ met.2013.0102)

8 Lopez-Garcia E, Guallar-Castillon P, Leon-Munoz L \& RodriguezArtalejo F. Prevalence and determinants of metabolically healthy obesity in Spain. Atherosclerosis 2013231 152-157. (https://doi. org/10.1016/j.atherosclerosis.2013.09.003)

9 Bell JA, Kivimaki M \& Hamer M. Metabolically healthy obesity and risk of incident type 2 diabetes: a meta-analysis of prospective cohort studies. Obesity Reviews 201415 504-515. (https://doi.org/10.1111/ obr.12157)

10 Hashimoto Y, Hamaguchi M, Tanaka M, Obora A, Kojima T \& Fukui M. Metabolically healthy obesity without fatty liver and risk of incident type 2 diabetes: a meta-analysis of prospective cohort studies. Obesity Research and Clinical Practice 201812 4-15. (https://doi.org/10.1016/j. orcp.2017.12.003)

11 Appleton SL, Seaborn CJ, Visvanathan R, Hill CL, Gill TK, Taylor AW, Adams RJ \& North West Adelaide Health Study Team. Diabetes and cardiovascular disease outcomes in the metabolically healthy obese phenotype: a cohort study. Diabetes Care 201336 2388-2394. (https:// doi.org/10.2337/dc12-1971)

12 Hinnouho GM, Czernichow S, Dugravot A, Nabi H, Brunner EJ, Kivimaki M \& Singh-Manoux A. Metabolically healthy obesity and the risk of cardiovascular disease and type 2 diabetes: the Whitehall II cohort study. European Heart Journal 201536 551-559. (https://doi. org/10.1093/eurheartj/ehu123)

13 Wang B, Zhang M, Wang S, Wang C, Wang J, Li L, Zhang L, Ren Y, Han C, Zhao Y et al. Dynamic status of metabolically healthy overweight/obesity and metabolically unhealthy and normal weight and the risk of type 2 diabetes mellitus: a cohort study of a rural adult Chinese population. Obesity Research and Clinical Practice 201812 61-71. (https://doi.org/10.1016/j.orcp.2017.10.005)

14 Luo D, Liu F, Li XW, Yin DC, Lin ZW, Liu H, Hou XH, Wang C \& Jia WP. Comparison of the effect of 'metabolically healthy but obese' and 'metabolically abnormal but not obese' phenotypes on development of diabetes and cardiovascular disease in Chinese. Endocrine 201549 130-138. (https://doi.org/10.1007/s12020-014-0444-2)

15 Eckel N, Li Y, Kuxhaus O, Stefan N, Hu FB \& Schulze MB. Transition from metabolic healthy to unhealthy phenotypes and association with cardiovascular disease risk across BMI categories in 90257 women (the Nurses' Health Study): 30 year follow-up from a prospective cohort study. Lancet: Diabetes and Endocrinology 20186 714-724. (https://doi.org/10.1016/S2213-8587(18)30137-2)

16 Mongraw-Chaffin M, Foster MC, Anderson CAM, Burke GL, Haq N, Kalyani RR, Ouyang P, Sibley CT, Tracy R, Woodward M et al. Metabolically healthy obesity, transition to metabolic syndrome, and cardiovascular risk. Journal of the American College of Cardiology 201871 1857-1865. (https://doi.org/10.1016/j.jacc.2018.02.055)

17 Chen Z, Lee L, Chen J, Collins R, Wu F, Guo Y, Linksted P \& Peto R. Cohort profile: the Kadoorie Study of Chronic Disease in China (KSCDC). International Journal of Epidemiology 200534 1243-1249. (https://doi.org/10.1093/ije/dyi174)

18 Chen ZM, Chen JS, Collins R, Guo Y, Peto R, Wu F, Li LM \& China Kadoorie Biobank (CKB) Collaborative Group. China Kadoorie biobank of 0.5 million people: survey methods, baseline characteristics and long-term follow-up. International Journal of Epidemiology 201140 1652-1666. (https://doi.org/10.1093/ije/dyr120)

19 Gao M, Lv J, Yu C, Guo Y, Bian Z, Yang R, Du H, Yang L, Chen Y, Li Z et al. Metabolically healthy obesity, transition to unhealthy metabolic status, and vascular disease in Chinese adults: a cohort study. PLoS Medicine 202017 e1003351. (https://doi.org/10.1371/journal. pmed.1003351)

20 Alberti KGMM, Eckel RH, Grundy SM, Zimmet PZ, Cleeman JI, Donato KA, Fruchart JC, James WPT, Loria CM, Smith SC et al. Harmonizing the metabolic syndrome: a joint interim statement of the International Diabetes Federation Task Force on Epidemiology and Prevention; National Heart, Lung, and Blood Institute; American Heart Association; World Heart Federation; International Atherosclerosis Society; and International Association for the Study of Obesity. Circulation 2009120 1640-1645. (https://doi.org/10.1161/ CIRCULATIONAHA.109.192644)

21 Chen C, Lu FC \& Department of Disease Control Ministry of Health, PR China. The guidelines for prevention and control of overweight and obesity in Chinese adults. Biomedical and Environmental Sciences 200417 (Supplement) 1-36.

22 Han Y, Hu Y, Yu C, Guo Y, Pei P, Yang L, Chen Y, Du H, Sun D, Pang Y et al. Lifestyle, cardiometabolic disease, and multimorbidity in a prospective Chinese study. European Heart Journal 202142 3374-3384. (https://doi.org/10.1093/eurheartj/ehab413)

23 Lv J, Qi L, Yu C, Yang L, Guo Y, Chen Y, Bian Z, Sun D, Du J, Ge P et al. Consumption of spicy foods and total and cause specific mortality: population based cohort study. BMJ 2015351 h3942. (https://doi. org/10.1136/bmj.h3942)

24 Plummer M. Improved estimates of floating absolute risk. Statistics in Medicine 200423 93-104. (https://doi.org/10.1002/sim.1485)

25 Fine JP \& Gray RJ. A proportional hazards model for the subdistribution of a competing risk. Journal of the American Statistical Association 199994 496-509. (https://doi.org/10.1080/01621459.1999. 10474144) 
26 Kramer CK, Zinman B \& Retnakaran R. Are metabolically healthy overweight and obesity benign conditions? A systematic review and meta-analysis. Annals of Internal Medicine 2013159 758-769. (https:// doi.org/10.7326/0003-4819-159-11-201312030-00008)

27 Lassale C, Tzoulaki I, Moons KGM, Sweeting M, Boer J, Johnson L, Huerta JM, Agnoli C, Freisling H, Weiderpass E et al. Separate and combined associations of obesity and metabolic health with coronary heart disease: a pan-European case-cohort analysis. European Heart Journal 201839 397-406. (https://doi.org/10.1093/eurheartj/ehx448)

28 WHO Expert Consultation. Appropriate body-mass index for Asian populations and its implications for policy and intervention strategies. Lancet 2004363 157-163. (https://doi.org/10.1016/S01406736(03)15268-3)

29 Caleyachetty R, Barber TM, Mohammed NI, Cappuccio FP, Hardy R, Mathur R, Banerjee A \& Gill P. Ethnicity-specific BMI cutoffs for obesity based on type 2 diabetes risk in England: a population-based cohort study. Lancet: Diabetes and Endocrinology 2021 9 419-426. (https://doi.org/10.1016/S2213-8587(21)00088-7)

30 Razak F, Anand SS, Shannon H, Vuksan V, Davis B, Jacobs R, Teo KK, McQueen M \& Yusuf S. Defining obesity cut points in a multiethnic population. Circulation 2007115 2111-2118. (https://doi.org/10.1161/ CIRCULATIONAHA.106.635011)

31 Caleyachetty R, Thomas GN, Toulis KA, Mohammed N, Gokhale KM, Balachandran K \& Nirantharakumar K. Metabolically healthy obese and incident cardiovascular disease events among 3.5 million men and women. Journal of the American College of Cardiology $2017 \mathbf{7 0}$ 1429-1437. (https://doi.org/10.1016/j.jacc.2017.07.763)

32 Stefan N, Schick F \& Haring HU. Causes, characteristics, and consequences of metabolically unhealthy normal weight in humans.
Cell Metabolism 201726 292-300. (https://doi.org/10.1016/j. cmet.2017.07.008)

33 Lotta LA, Gulati P, Day FR, Payne F, Ongen H, van de Bunt M, Gaulton KJ, Eicher JD, Sharp SJ, Luan J et al. Integrative genomic analysis implicates limited peripheral adipose storage capacity in the pathogenesis of human insulin resistance. Nature Genetics 201749 17-26. (https://doi.org/10.1038/ng.3714)

34 Eigentler T, Lomberg D, Machann J \& Stefan N. Lipodystrophic nonalcoholic fatty liver disease induced by immune checkpoint blockade. Annals of Internal Medicine 2020172 836-837. (https://doi. org/10.7326/L19-0635)

35 Stefan N. Causes, consequences, and treatment of metabolically unhealthy fat distribution. Lancet: Diabetes and Endocrinology 20208 616-627. (https://doi.org/10.1016/S2213-8587(20)30110-8)

36 Stefan N, Häring HU \& Schulze MB. Metabolically healthy obesity: the low-hanging fruit in obesity treatment? Lancet: Diabetes and Endocrinology 20186 249-258. (https://doi.org/10.1016/S2213-8587(17)30292-9)

37 Phillips CM. Metabolically healthy obesity across the life course: epidemiology, determinants, and implications. Annals of the New York Academy of Sciences 20171391 85-100. (https://doi.org/10.1111/ nyas.13230)

38 Bell JA, Hamer M, Sabia S, Singh-Manoux A, Batty GD \& Kivimaki M. The natural course of healthy obesity over 20 years. Journal of the American College of Cardiology 201565 101-102. (https://doi. org/10.1016/j.jacc.2014.09.077)

39 Navarro-Gonzalez D, Sanchez-Inigo L, Fernandez-Montero A, Pastrana-Delgado J \& Martinez JA. Are all metabolically healthy individuals with obesity at the same risk of diabetes onset? Obesity 201624 2615-2623. (https://doi.org/10.1002/oby.21667)

Received 24 July 2021

Revised version received 12 November 2021

Accepted 7 December 2021 